\title{
The contribution of ion channels in input-output plasticity
}

\author{
Dominique Debanne*, Michaël Russier \\ UNIS, UMR1072 INSERM - AMU, Marseille, France
}

\section{A R T I C L E I N F O}

\section{Keywords:}

Ion channels

Activity-dependent plasticity

Learning

Memory

LTP

LTD

STDP

\begin{abstract}
A B S T R A C T
Persistent changes that occur in brain circuits are classically thought to be mediated by long-term modifications in synaptic efficacy. Yet, many studies have shown that voltage-gated ion channels located at the input and output side of the neurons are also the subject to persistent modifications. These channels are thus responsible for intrinsic plasticity that is expressed in many different neuronal types including glutamatergic principal neurons and GABAergic interneurons. As for synaptic plasticity, activation of synaptic glutamate receptors initiate persistent modification in neuronal excitability. We review here how synaptic input can be efficiently altered by activity-dependent modulation of ion channels that control EPSP amplification, spike threshold or resting membrane potential. We discuss the nature of the learning rules shared by intrinsic and synaptic plasticity, the mechanisms of ion channel regulation and the impact of intrinsic plasticity on induction of synaptic modifications.
\end{abstract}

\section{Introduction}

Information storage in the brain is classically thought to result from long-lasting modifications of chemical synaptic transmission. Support to this has been provided by many studies showing up- or down-regulation of synaptic transmission following specific regimes of synaptic activity. For instance, long-term synaptic potentiation (LTP) occurs following high frequency stimulation of afferent glutamatergic inputs at many synaptic pathways including the Schaffer collateral-CA1 cell synapse (Bliss \& Collingridge, 1993; Malenka, 1991), mossy-fibre-CA3 cell synapse (Zalutsky \& Nicoll, 1990), visual neocortical inputs (Artola \& Singer, 1987), and glutamatergic afferents to inhibitory interneurons (Lamsa, Heeroma, Somogyi, Rusakov, \& Kullmann, 2007). Long-term synaptic depression (LTD) is induced homosynaptically following low frequency stimulation of glutamatergic inputs. Evidence for homosynaptic LTD has been provided at the Schaffer collateral-CA1 cell synapse (Dudek \& Bear, 1992; Mulkey \& Malenka, 1992), inputs to visual cortical neurons (Kirkwood, Dudek, Gold, Aizenman, \& Bear, 1993). LTP and LTD have been shown to be induced associatively when a synaptic input is paired with postsynaptic spiking activity. LTP is induced at Schaffer collateral inputs when glutamatergic input is synchronously paired with burst of action potentials (Gustafsson, Wigstrom, Abraham, \& Huang, 1987; Kelso, Ganong, \& Brown, 1986; Sastry, Goh, \& Auyeung, 1986) whereas LTD results of asynchronous activation of these two stimuli (Debanne et al., 1994, 1996). These forms of associative synaptic plasticity led to the emergence of the spike-timing- dependent plasticity (STDP) rule (Abbott \& Nelson, 2000; Feldman, 2012) in which positive correlation between excitatory post-synaptic potentials (EPSPs) and single spikes led to LTP whereas their negative correlation led to LTD (Bi \& Poo, 1998; Debanne, Gahwiler, \& Thompson, 1998; Feldman, 2000; Markram, Lubke, Frotscher, \& Sakmann, 1997).

All these synaptic modifications are specific to the synapse stimulated during the cellular conditioning (Bliss \& Collingridge, 1993; Debanne, Gahwiler, \& Thompson, 1994; Malenka, 1991) and may thus allow high capacity of information storage. However, synaptic plasticity appears to not constitute the sole manner to modify input-output function and voltage-gated channels located at the input and output sides of neurons are also subject to activity-dependent modification (Debanne, Inglebert, \& Russier, 2019; Titley, Brunel, \& Hansel, 2017; Zhang \& Linden, 2003). Here, we review recent in vitro works devoted to the role of ion channels in the modulation of input-output function in mammalian neurons. First, we will consider the different ways of modulating input-output function at the neuronal scale. Then, we will review the learning rules of input-output plasticity on the basis of those defined for synaptic modifications. Finally, we will discuss intrinsic plasticity in GABAergic interneurons, the impact of ion channel regulation on induction of synaptic plasticity and intrinsic plasticity in vivo.

\section{Activity-dependent modulation in input-output function}

Input-output function is a critical operation at the neuronal scale

\footnotetext{
* Corresponding author.

E-mail address: dominique.debanne@inserm.fr (D. Debanne).
} 
A

Synaptic plasticity

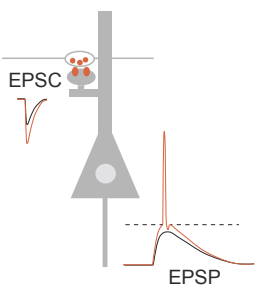

C

Threshold modulation

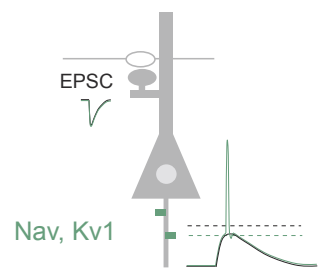

Fig. 1. Multiple mechanisms of input-output plasticity. (A) Synaptic contribution to input-output modulation. Potentiation of synaptic transmission through an increase in transmitter release and/or postsynaptic receptor density is characterized by an enhanced excitatory post-synaptic current (EPSC). At the axon initial segment (AIS), the excitatory post-synaptic potential (EPSP) becomes large enough to cross the action potential (AP) threshold. As synaptic strength is regulated, EPSP slope is enhanced. (B) Change in EPSP amplification. When dendritic HCN or Kv4 channels (green) are regulated, the resulting EPSP is amplified and crosses the spike threshold. Note that here the synaptic current (EPSC) is kept constant to clearly distinguish intrinsic from synaptic changes and the initial EPSP slope remains unchanged. These first two modifications (A \& B) are local because they do not affect all synaptic inputs. (C) Change in spike threshold. Spike firing increases as a result of hyperpolarization of AP threshold by the regulation of voltage-gated ion channels (Nav or Kv1) located at the AIS. Here again, the excitatory synaptic current (EPSC) remains unchanged. (D) Depolarization of resting membrane potential. Following modulation of voltage-gated channels (HCN) or ion pumps (Na/K ATPase), the resting membrane potential of the neuron is depolarized. Thus, an action potential is now trigged by the EPSP. The synaptic current (EPSC) remains unchanged. These last two modifications (C \& D) are global because they may affect all synaptic inputs. (For interpretation of the references to colour in this figure legend, the reader is referred to the web version of this article.)

that is achieved by synaptic and intrinsic mechanisms. Whereas expression mechanisms of synaptic plasticity are well identified today and involve either presynaptic change in neurotransmitter release or postsynaptic change in glutamate receptor density or function (Fig. 1A), modulation of input-output function can be achieved through at least 3 different types of functional modulation: change in EPSP amplification (Fig. 1B), spike threshold modulation (Fig. 1C) and membrane potential adjustment (Fig. 1D) (Debanne et al., 2019). In addition, modulation of the balance between synaptic inhibition and excitation is responsible for apparent input-output plasticity that occurs following induction of LTP (Lu, Mansuy, Kandel, \& Roder, 2000; Orr et al., 2014) or LTD (Kim, Park, Lee, \& Ho, 2019).

\subsection{Control of EPSP amplification}

Changes in EPSP amplification represent a powerful mean to alter input-output function in central neurons. Ion channels located in dendrites and in the cell body are activated by EPSPs (Magee, 2000; Shah, 2014; Spruston, 2008). As a consequence, they shape EPSP waveform by either boosting or attenuating the synaptic response (Fig. 1B). A good example for this is provided by hyperpolarization-activated cyclic nucleotide-gated (HCN) cationic channels responsible for the h-current
(Magee, 1999). These channels are activated at resting membrane potential and attenuate EPSP amplitude through their deactivation. Thus, any modulation of h-channels may increase or decrease EPSP-spike coupling (Gastrein et al., 2011). The down-regulation of these channels have been shown to enhance EPSP summation (Wang, Xu, Wu, Duan, \& Poo, 2003) and input-output function (Campanac, Daoudal, Ankri, \& Debanne, 2008) following LTP induction. Kv4.2 is another voltagegated ion channel located in the dendrites that attenuates both EPSP amplitude and back-propagating action potentials in distal dendrites (Hoffman, Magee, Colbert, \& Johnston, 1997). The down-regulation of Kv4.2 channel activity results in enhancement of both EPSP amplitude and local back-propagation (Frick, Magee, \& Johnston, 2004; Kim, Jung, Clemens, Petralia, \& Hoffman, 2007). As synaptic plasticity, the modulation of EPSP amplification is generally local as other inputs remain unchanged (Campanac \& Debanne, 2008; Wang et al., 2003). EPSPs are also shaped by small conductance calcium-activated $\mathrm{K}^{+}$(SK) channels. Calcium influx through NMDAR has been shown to activate SK channels located in the dendritic spines thus attenuating EPSP amplitude and duration (Adelman, Maylie, \& Sah, 2012; Faber, Delaney, \& Sah, 2005; Ngo-Anh et al., 2005). Interestingly, SK channels are downregulated following stimulation of synaptic metabotropic glutamate receptor subtype 5 (mGluR5) resulting in enhancement of EPSPspike coupling (Sourdet, Russier, Daoudal, Ankri, \& Debanne, 2003). Nevertheless, EPSP-spike coupling in cortical neurons is poorly determined by EPSP amplitude and highly dependent on the rate and the waveform of dendritic EPSPs (Larkum, Zhu, \& Sakmann, 2001). In cerebellar Purkinje cells, EPSP amplitude has also limited control over cell firing but pharmacological modulation of SK channel and SK-dependent plasticity strongly regulate spike firing (Ohtsuki \& Hansel, 2018).

\subsection{Modulation of spike threshold}

Input-output function may also be altered via modulation of ion channels that control the spike threshold (Fig. 1C). Voltage-gated $\mathrm{Na}^{+}$ (Nav) and $\mathrm{K}^{+}(\mathrm{Kv})$ channels determine the spike threshold (Bean, 2007). Shift of Nav activation towards hyperpolarized values lowers the spike threshold and increases excitability following induction of longterm synaptic potentiation in CA1 pyramidal neurons (Xu, Kang, Jiang, Nedergaard, \& Kang, 2005). Down-regulation of Kv1 channels following chronic activity-deprivation with pharmacological treatment in hippocampal neurons (Cudmore, Fronzaroli-Molinieres, Giraud, \& Debanne, 2010) or following cochlea removal in auditory neurons (Kuba, Yamada, Ishiguro, \& Adachi, 2015), lowers the spike threshold and increases intrinsic excitability. It should be noted that in contrast to change in EPSP amplification, spike-threshold modulation is global since it may affect all incoming inputs.

\subsection{Modification of resting membrane potential}

Change in resting membrane potential (RMP) represents the third manner to modulate input-output function by non-synaptic mechanisms (Fig. 1D). In hippocampal granule cells, high frequency firing induces long-term depolarization (LT-Depol) of their RMP by approximately 8-10 mV (Mellor, Nicoll, \& Schmitz, 2002) that is mediated by a protein kinase A-dependent up-regulation of HCN channels. Whereas the upregulation of HCN channels leads to attenuated EPSP amplitude and therefore to a reduction in intrinsic excitability (see above), the net effect here is however an increased excitability. In fact, the large depolarization of RMP $(8-10 \mathrm{mV})$ largely dominates the excitability reduction caused by the attenuation of excitatory synaptic inputs. Here again, this modulation is global as all inputs will be equally affected. 


\section{Synaptic transmission}

\section{A}

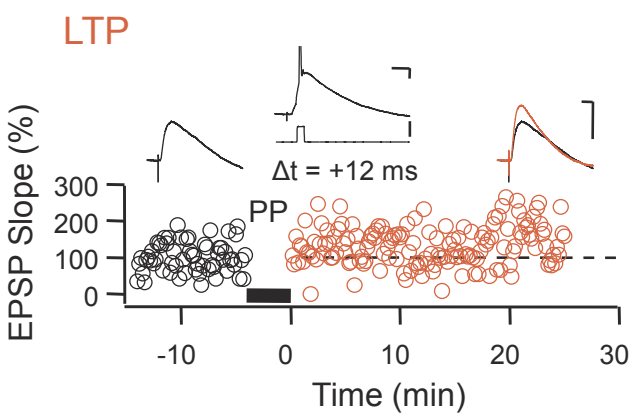

B

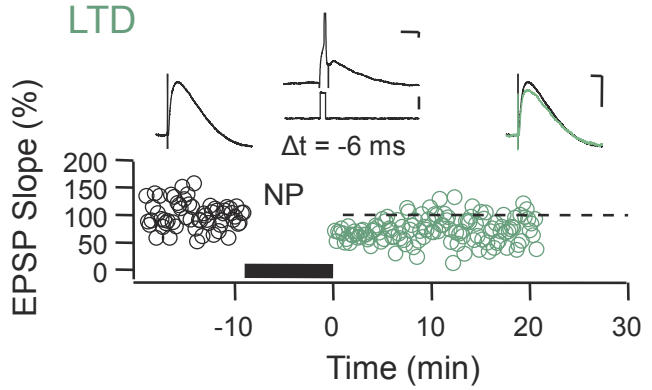

C

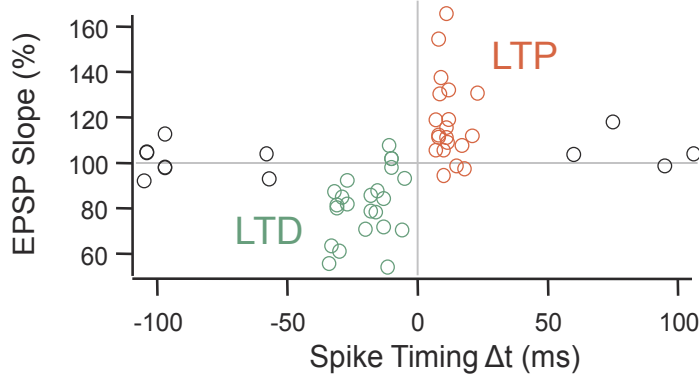

Excitability
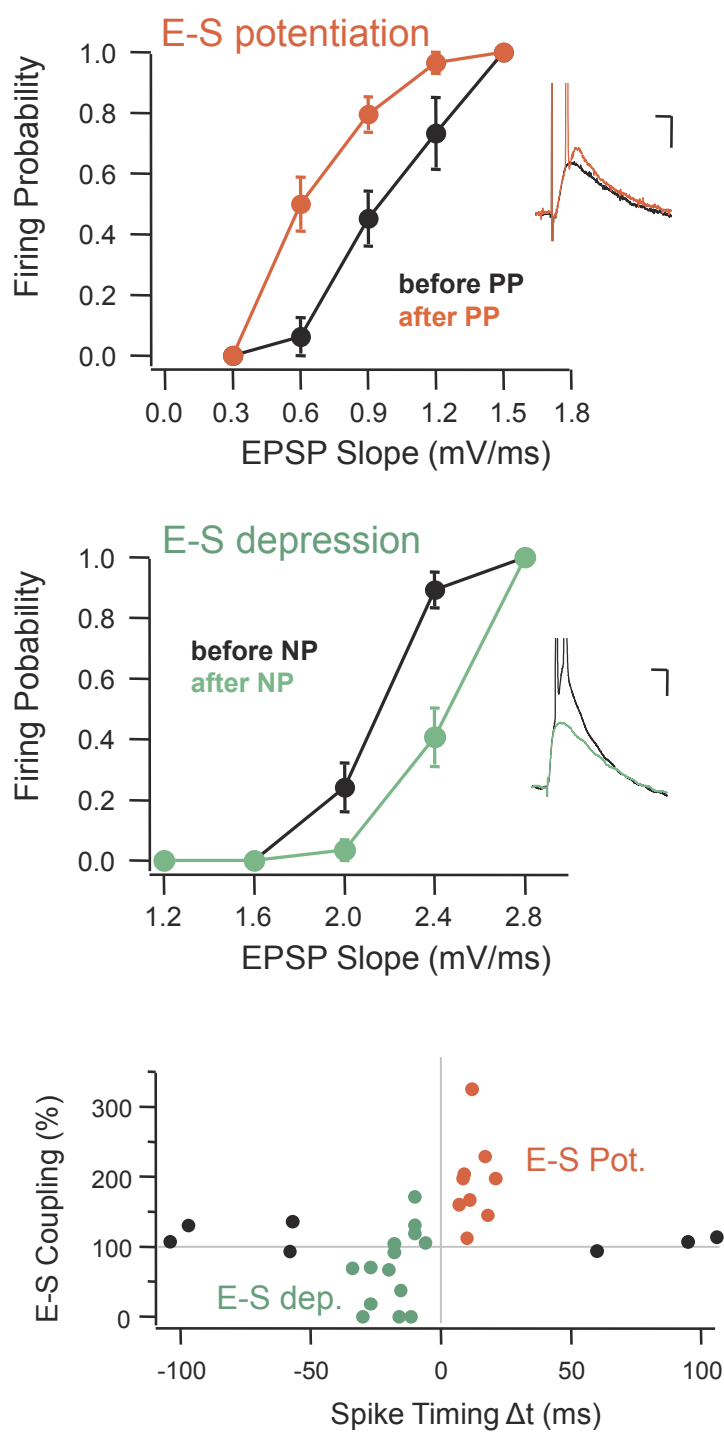

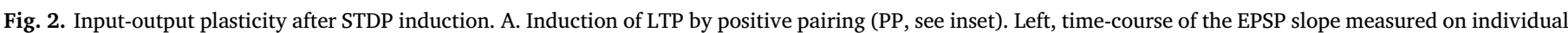

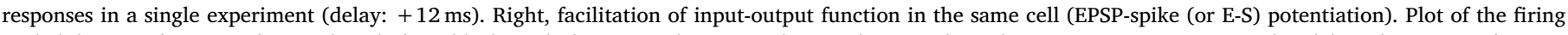

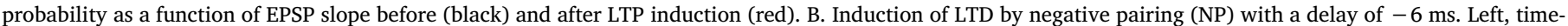

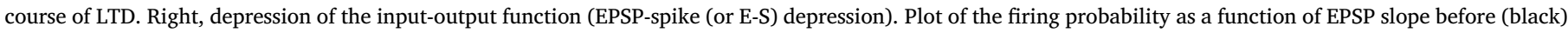

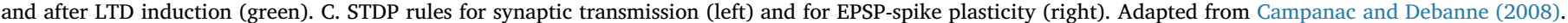
(For interpretation of the references to colour in this figure legend, the reader is referred to the web version of this article.)

\section{Learning rules for input-output plasticity}

\subsection{Hebbian changes in input-output function}

Hebbian synaptic plasticity represents the better described forms of synaptic modifications. According to the Bienenstock Cooper \& Munro rule (Bienenstock, Cooper, \& Munro, 1982), it can be induced homosynaptically by modulation of synaptic frequency (Bear, 1996; Cooper \& Bear, 2012). Hebbian synaptic modification in the hippocampus and neocortex can also be induced by the degree of correlation between preand postsynaptic activity and is referred to as spike-timing dependent plasticity or STDP (Feldman, 2012). Both forms of Hebbian plasticity are associated with intrinsic plasticity that are synergistic to the induced synaptic changes (Debanne et al., 2019).

In CA1 pyramidal neurons, long-term synaptic potentiation (LTP) is associated with an increased firing probability in response to the tetanized or paired input (Fig. 2A) whereas long-term synaptic depression (LTD) is associated with a decreased firing probability (Fig. 2B) in response to the stimulated input (Campanac \& Debanne, 2008; Daoudal, Hanada, \& Debanne, 2002). Interestingly, the STDP rule defined for synaptic transmission also applies to EPSP-spike coupling (Fig. 2C). These modification in EPSP-spike coupling are supposed to result from changes in EPSP amplification. In fact, EPSP summation is altered in a synergistic manner with synaptic modifications induced by STDP protocols (Wang et al., 2003). Most Hebbian modifications in input-output function require NMDA-receptor activation for their induction and are input-specific, i.e. no modification occurs on control inputs. More recently, brief episodes of firing at $10 \mathrm{~Hz}$ in CA3 pyramidal neurons have been shown to enhance input resistance and integration of distal EPSP (Hyun et al., 2015). Hebbian intrinsic plasticity is not specific to hippocampal neurons and long-lasting increase in intrinsic excitability (IE) has been reported in neocortical neurons following 

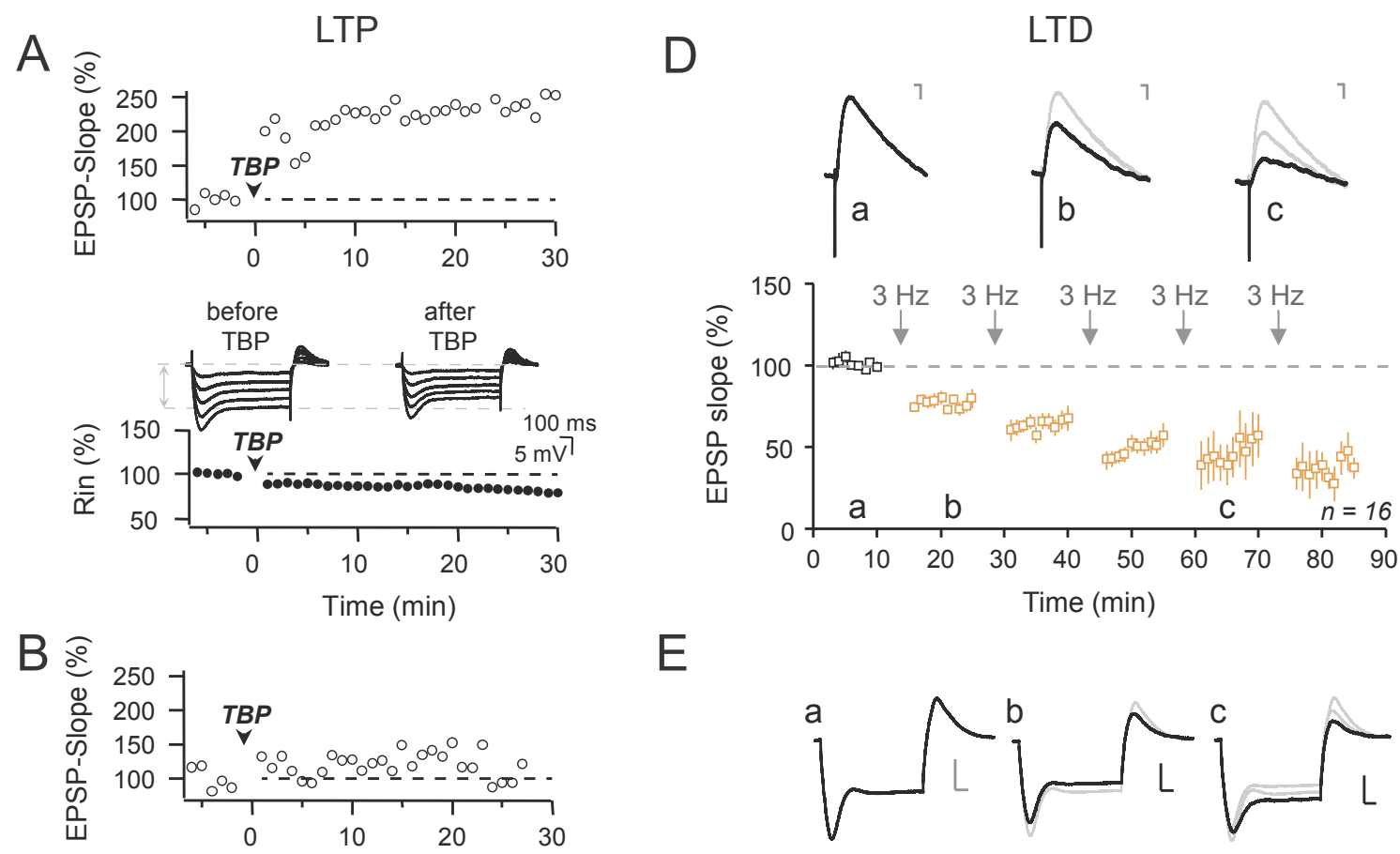

$E$
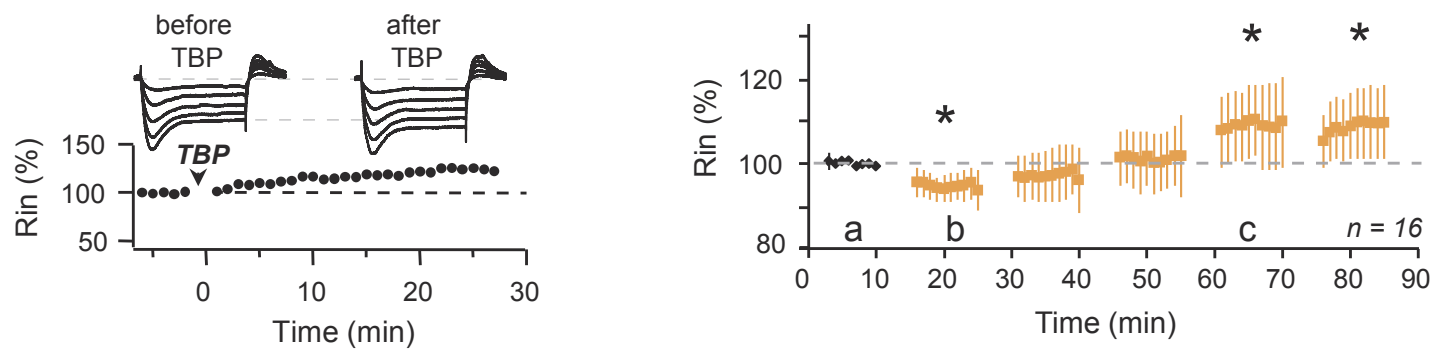

C

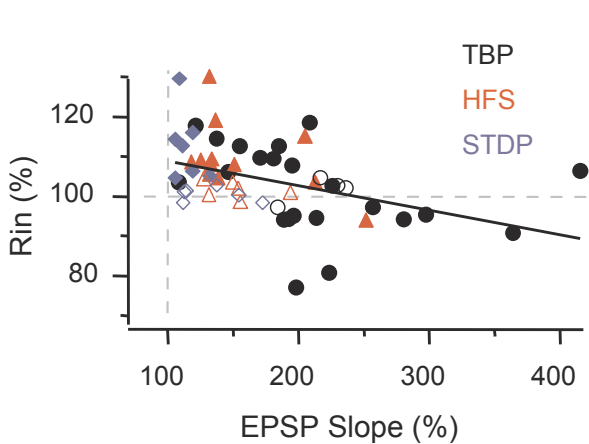

F

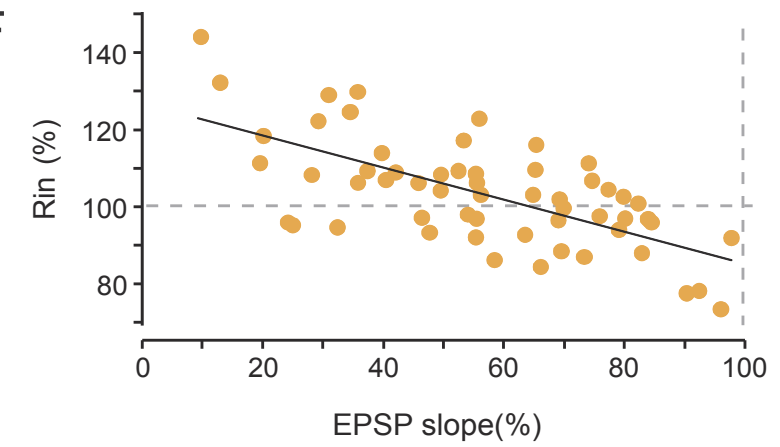

$G$

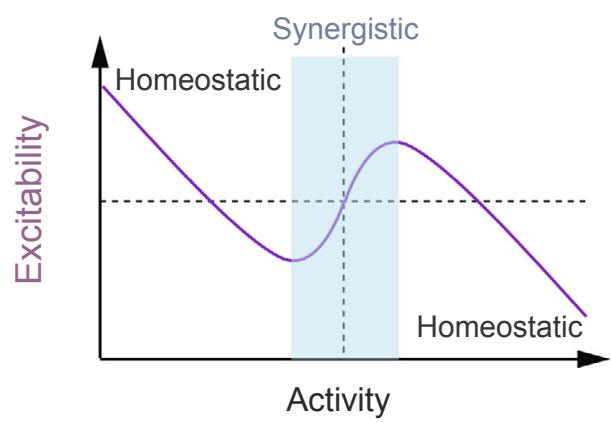

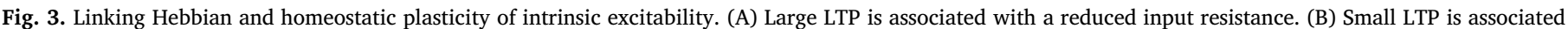

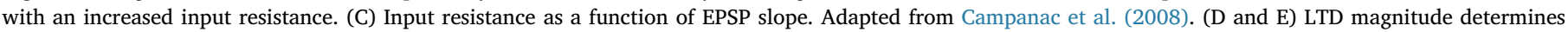

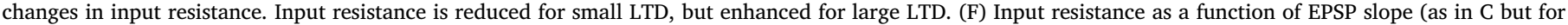

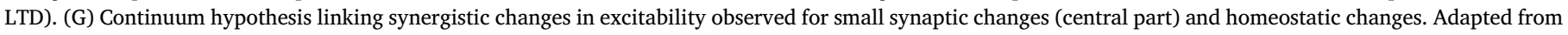
Gasselin et al. (2017). 
synaptic (Desai, Casimiro, Gruber, \& Vanderklish, 2006; Sourdet et al., 2003) or intrinsic (Cudmore \& Turrigiano, 2004; Mahon \& Charpier, 2012; Paz et al., 2009) activation paradigms.

Hebbian intrinsic plasticity is also observed in cerebellar neurons. In deep cerebellar nuclei, granule and Purkinje neurons, IE was found to be enhanced in parallel of LTP induced by high frequency stimulation (Aizenman \& Linden, 2000; Armano, Rossi, Taglietti, \& D'Angelo, 2000; Belmeguenai et al., 2010). In all these cases, induction of intrinsic plasticity requires NMDA-receptor activation. Expression mechanisms in cerebellar neurons involve regulation of SK channels (Belmeguenai et al., 2010; Ohtsuki, Piochon, Adelman, \& Hansel, 2012) and HCN channels (Shim et al., 2017).

\subsection{Homeostatic plasticity of input-output function}

Hebbian mechanisms are destabilizing as they tend to reinforce active circuits and depress inactive ones. Neural circuits can be stabilized when homeostatic regulation is introduced to adjust both synaptic strength and intrinsic excitability (Turrigiano \& Nelson, 2004). Homeostatic plasticity of IE has been originally reported in visual cortical neurons (Desai, Rutherford, \& Turrigiano, 1999b) and is today observed in virtually all neuronal types. Chronic activity deprivation induced pharmacologically (Cudmore et al., 2010; Gasselin, Inglebert, \& Debanne, 2015; Karmarkar \& Buonomano, 2006; Shim et al., 2016) or by sensory deprivation (Kuba et al., 2015; Maffei \& Turrigiano, 2008; Milshtein-Parush et al., 2017) enhances IE. Reduced IE is observed following elevated network activity (Gasselin et al., 2015; Karmarkar \& Buonomano, 2006; Kirchheim, Tinnes, Haas, Stegen, \& Wolfart, 2013). Thus, homeostatic plasticity rule is per se anti-Hebbian.

Two ion channels that dampen excitability have been particularly studied in homeostatic regulation of IE: (1) Kv1 channels located in axons of pyramidal neurons which determine spike threshold and intrinsic excitability (Rama et al., 2017), and (2) HCN channels located in the dendrites that dampens all depolarizing events such as EPSPs (Magee, 1999). Down-regulation of Kv1 channel activity has been identified as a major mechanism for the increased excitability observed in CA3 pyramidal neurons after chronic blockade of glutamate receptors (Cudmore et al., 2010) and in auditory neurons following cochlear removal (Kuba et al., 2015). Conversely, Kv1 channel activity is up-regulated in dentate granule cells following epileptiform activity (Kirchheim et al., 2013), indicating that Kv1 channel activity can be bidirectionally adjusted to maintain constant circuit activity. In CA1 pyramidal neurons, h-current is homeostatically regulated following induction of extremely large synaptic modifications (Brager \& Johnston, 2007; Fan et al., 2005) or following bidirectional chronic manipulation of network activity (Gasselin et al., 2015).

Homeostatic plasticity is typically induced by persistent modulation of activity lasting few tens of hours (Cudmore et al., 2010; Desai et al., 1999b; Gasselin et al., 2015; Karmarkar \& Buonomano, 2006). However, homeostatic increase in excitability can also be induced by brief stimulation. For instance, in vestibular neurons (Nelson, Krispel, Sekirnjak, \& du Lac, 2003) and cerebellar Golgi cells (Hull, Chu, Thanawala, \& Regehr, 2013), a transient hyperpolarization $(20-300 \mathrm{sec})$ results in enhanced IE. In both cases, potentiation of IE requires the down-regulation of BK channel activity. One may notice that in contrast to cortical pyramidal cells that are mostly silent at rest, vestibular or cerebellar neurons correspond to pacemaker cells that fire continuously at frequency ranging between 5 and $10 \mathrm{~Hz}$. Recently, it has been shown that unilateral loss of inner hear triggers rapid potentiation of excitability in vestibular neurons thus enabling adaptive compensatory increases in optokinetic reflex gain (Nelson et al., 2017).

\subsection{How can Hebbian and homeostatic intrinsic plasticity be linked together?}

Theoretical work suggests that Hebbian and homeostatic plasticity, despite their antagonistic rules, may work hand-in-hand (Zenke \& Gerstner, 2017). The modulation of HCN channels following induction of synaptic modification provides a good example for such complex interaction. While large LTP $(>+200 \%)$ is associated with decreased IE due to the up-regulation of HCN channel activity (Fan et al., 2005) (Fig. 3A), small LTP $(<+100 \%)$ is combined with increased IE resulting from down-regulation of HCN channel (Campanac \& Debanne, 2008) (Fig. 3B and C). The opposite holds true for LTD. Large LTD $(<-50 \%)$ results in a HCN-dependent increase in IE (Brager \& Johnston, 2007) whereas small LTD $(>-30 \%)$ results in a HCN-dependent decrease in IE (Gasselin, Inglebert, Ankri, \& Debanne, 2017) (Fig. 3D-F). Taken together, these results indicate that IE follows a single plasticity rule linking Hebbian and homeostatic plasticity (Fig. 3G). This multiple regulation of HCN channel implies distinct induction and expression pathways (Brager \& Johnston, 2007; Fan et al., 2005; Gasselin et al., 2017; Santoro et al., 2009).

\section{Activity-dependent regulation of excitability in GABAergic interneurons}

Principal neurons are not the only cell type to express intrinsic plasticity. GABAergic interneurons also display several forms of longterm regulation of IE. High-frequency stimulation of the glutamatergic inputs to dentate basket cells induces LT-Depol of their resting membrane potential that results from calcium-permeable AMPA receptordependent modulation of the $\mathrm{Na}^{+} / \mathrm{K}^{+}$ATPase pump (Ross \& Soltesz, 2001). Inhibition levels in cortical circuits have been shown to be finely tuned through Kv1-dependent modulation of the spike threshold in basket cells. Neuregulin 1 receptor ErbB4 strongly regulates Kv1 channel activity and intrinsic excitability in parvalbumin positive basket cells (PV-BC) (Li et al., 2011). Synaptic stimulation of mGluR5 induces long-lasting potentiation of IE in PV-BCs that is mediated by the down-regulation of Kv1 channel activity (Campanac et al., 2013). Enhanced excitability in PV-BCs is due to hyperpolarized spike threshold and is thought to compensate enhanced synaptic and intrinsic excitation in pyramidal neurons. Interestingly, the reciprocal modulation of IE has been reported in somatosensory PV interneurons following activity-deprivation (Gainey, Aman, \& Feldman, 2018; Sun, 2009). Thus, this suggests that Kv1-dependent regulation of neuronal excitability is bidirectional in PV-BC. Most cortical PV interneurons express Er81, a transcription factor highly regulated by activity and involved in the activation pathway of $\mathrm{Ca}^{2+}$ /calmodulin-dependent kinase I and in the control of levels of Kv1.1 channels in PV interneurons (Dehorter et al., 2015). Thus, Er81 level is high in weakly active circuits (corresponding to high levels of Kv1.1 channels) whereas it is low in highly active circuits (corresponding to low levels of Kv1.1 channels). Reduced input resistance has also been reported in fast-spiking interneurons from the dentate gyrus following synaptic stimulations of the mossy-fibers (Dasgupta \& Sikdar, 2015). This modulation is mediated by HCN channels and depends on activation of calcium-permeable AMPA receptor. Computer simulations indicate that intrinsic plasticity in interneurons robustly modulates both the coherence and frequency of network activity (Dasgupta \& Sikdar, 2019).

\section{Consequences of input-output intrinsic plasticity on induction of synaptic plasticity}

Regulation of dendritic conductance may alter induction of synaptic plasticity. For instance, the homeostatic regulation of h-channels not only modulates EPSP summation (Gasselin et al., 2015; Shah, Anderson, Leung, Lin, \& Johnston, 2004) but also stabilizes synaptic plasticity rules (Gasselin et al., 2015; Mishra \& Narayanan, 2015). Furthermore, the down-regulation of SK channel activity as shown to occur following synaptic activation of mGluR5 (Sourdet et al., 2003) facilitates LTP induction at cortical (Faber et al., 2005; Jones, To, \& Stuart, 2017) and hippocampal (Tigaret et al., 2018) synapses. Finally, the down- 
regulation of Kv4 channel activity observed following induction of LTP facilitates locally the back-propagation of the action potential (Frick et al., 2004) and may help to induce LTP associatively at neighboring synapses (Chen et al., 2006).

\section{Molecular mechanisms of input-output plasticity}

Expression mechanisms of synaptic plasticity have been extensively studied over the last 30 years and revealed several key features. First, glutamate receptors present at the synapse are phosphorylated by many protein kinases such as CaMKII (Herring \& Nicoll, 2016; Lisman, Yasuda, \& Raghavachari, 2012) or PKC (Sacktor \& Hell, 2017). Other kinases such as PKA and mTOR are involved respectively in presynaptic LTP (Huang et al., 1995) and in induction of BDNF-dependent LTP (Tang et al., 2002). Glutamate receptors show a gain in their function when they are phosphorylated (i.e. they generate a larger current upon activation by glutamate). Two protein phosphatases, PP1 or PP2 (calcineurin), occupy a critical position in the induction of synaptic LTD (Lisman, 1989; Winder \& Sweatt, 2001). Second, synaptic receptors are mobile within the dendritic spine (Herring \& Nicoll, 2016) and insertion of AMPA receptors have been shown to represent a major way to enhance synaptic efficacy (Penn et al., 2017).

Many of the key molecular actors identified in synaptic plasticity are also found in intrinsic plasticity. For instance, the phosphorylation of Kv4.2 by PKA and PKC not only reduces the current by shifting activation curve of the A-current (Hoffman \& Johnston, 1998) but it also facilitates internalization of Kv4.2 (Kim et al., 2007), both leading to a loss of function of these channels. CaMKII phosphorylation is required for the homeostatic modulation of HCN channels following LTP induction (Fan et al., 2005). BDNF determines homeostatic plasticity of intrinsic excitability (Desai, Rutherford, \& Turrigiano, 1999a). Regulation of Kv1 channels has been shown to depend on mTOR (Campanac et al., 2013; Raab-Graham, Haddick, Jan, \& Jan, 2006). Calcineurin is involved in both the homeostatic dispersion of Kv2.1 (Misonou et al., 2004) following chronic excitation and the relocation of the axon initial segment (Evans et al., 2013).

\section{Input-output intrinsic plasticity in vivo}

Most of the recent advances in understanding induction and expression mechanisms of intrinsic plasticity come from in vitro studies. A large bundle of papers has been devoted to the search for changes in cellular excitability correlated with learning and memory in the mammalian brain. Learning-driven changes in neuronal excitability have been reported following classical conditioning, fear conditioning or spatial exploration in hippocampal, cerebellar and cortical neurons. Active neurons during conditioning display in vitro excitability in conditioned animals that is significantly higher than in naïve or pseudoconditioned animals (Disterhoft, Coulter, \& Alkon, 1986; Oh, Kuo, Wu, Sametsky, \& Disterhoft, 2003; Santini, Quirk, \& Porter, 2008; Schreurs, Tomsic, Gusev, \& Alkon, 1997). Because of population analysis used in most of these studies, changes in excitability of physiologically characterized neurons in vivo remain uncertain.

The gap has been filled more recently with studies of intrinsic excitability in single cortical neurons before and after cellular conditioning (Mahon \& Charpier, 2012; Paz et al., 2009). Increased or decreased excitability has been observed in rat motor cortical neurons following repeated bursting at $30 \mathrm{~Hz}$ (Paz et al., 2009) or at $10 \mathrm{~Hz}$ (Mahon \& Charpier, 2012). These regulation in excitability involved either shift in input-output curves, or modulation of spike-threshold by $\sim 2 \mathrm{mV}$ (Mahon \& Charpier, 2012). Interestingly, the polarity of the intrinsic change was related to the neuron firing rate in control conditions (Mahon \& Charpier, 2012), suggesting that intrinsic changes rather act as a homeostatic mechanism.

\section{Conclusion and perspective}

Synaptic plasticity has been extensively studied over the last 30 years but it appears to represent only a portion of functional plasticity. Striking progress has been made these recent years in understanding learning rules and in identifying expression mechanisms of intrinsic plasticity. Intrinsic plasticity is bidirectional, induced in parallel to synaptic plasticity and has been shown to be expressed at nearly all cellular types. Many different ion channels involved in intrinsic plasticity have been identified. But many questions are still pending. For example, most if not all studies reported in this review comes from in vitro works. Only a handful of in vivo studies devoted to intrinsic plasticity has been reported with physiologically realistic induction protocols (Mahon \& Charpier, 2012; Paz et al., 2009). Further studies must be performed to monitor IE in cortical or cerebellar neurons during the acquisition of a simple behavioral task. To reach this aim, new methods and experimental tools will have to be developed. Synaptic and intrinsic changes occur often in parallel. Dissection of the respective contribution of synaptic and intrinsic plasticity in the modulation of synaptic circuits is another challenge for the next years that will require development of new strategies. What are the mechanisms behind the tight link between synaptic and intrinsic changes? New imaging methods will have to be developed to simultaneously follow the trafficking of synaptic receptors and ion channels following induction of plasticity. The future will probably help to answer all these questions.

\section{Acknowledgments}

Supported by INSERM, CNRS, AMU and FRM (DVS20131228768 \& DEQ2018083983). We are indebted to Emilie Campanac, Celia Gasselin \& Yanis Inglebert for their contribution in the original work on which is based this review paper.

\section{References}

Abbott, L. F., \& Nelson, S. B. (2000). Synaptic plasticity: Taming the beast. Nature Neuroscience, 3(Suppl), 1178-1183.

Adelman, J. P., Maylie, J., \& Sah, P. (2012). Small-conductance $\mathrm{Ca}^{2+}$-activated K+ channels: Form and function. Annual Review of Physiology, 74, 245-269.

Aizenman, C. D., \& Linden, D. J. (2000). Rapid, synaptically driven increases in the intrinsic excitability of cerebellar deep nuclear neurons. Nature Neuroscience, 3, $109-111$.

Armano, S., Rossi, P., Taglietti, V., \& D'Angelo, E. (2000). Long-term potentiation of intrinsic excitability at the mossy fiber-granule cell synapse of rat cerebellum. Journal of Neuroscience, 20, 5208-5216.

Artola, A., \& Singer, W. (1987). Long-term potentiation and NMDA receptors in rat visual cortex. Nature, 330, 649-652.

Bean, B. P. (2007). The action potential in mammalian central neurons. Nature Reviews Neuroscience, 8, 451-465.

Bear, M. F. (1996). A synaptic basis for memory storage in the cerebral cortex. Proceedings of the National Academy of Sciences of the United States of America, 93, 13453-13459.

Belmeguenai, A., Hosy, E., Bengtsson, F., Pedroarena, C. M., Piochon, C., Teuling, E., Hansel, C. (2010). Intrinsic plasticity complements long-term potentiation in parallel fiber input gain control in cerebellar Purkinje cells. Journal of Neuroscience, 30, 13630-13643.

Bi, G. Q., \& Poo, M. M. (1998). Synaptic modifications in cultured hippocampal neurons: Dependence on spike timing, synaptic strength, and postsynaptic cell type. Journal of Neuroscience, 18, 10464-10472.

Bienenstock, E. L., Cooper, L. N., \& Munro, P. W. (1982). Theory for the development of neuron selectivity: Orientation specificity and binocular interaction in visual cortex. Journal of Neuroscience, 2, 32-48.

Bliss, T. V., \& Collingridge, G. L. (1993). A synaptic model of memory: Long-term potentiation in the hippocampus. Nature, 361, 31-39.

Brager, D. H., \& Johnston, D. (2007). Plasticity of intrinsic excitability during long-term depression is mediated through mGluR-dependent changes in I(h) in hippocampal CA1 pyramidal neurons. Journal of Neuroscience, 27, 13926-13937.

Campanac, E., Daoudal, G., Ankri, N., \& Debanne, D. (2008). Downregulation of dendritic I(h) in CA1 pyramidal neurons after LTP. Journal of Neuroscience, 28, 8635-8643.

Campanac, E., \& Debanne, D. (2008). Spike timing-dependent plasticity: A learning rule for dendritic integration in rat CA1 pyramidal neurons. Journal of Physiology, 586, 779-793.

Campanac, E., Gasselin, C., Baude, A., Rama, S., Ankri, N., \& Debanne, D. (2013). Enhanced intrinsic excitability in basket cells maintains excitatory-inhibitory balance in hippocampal circuits. Neuron, 77, 712-722. 
Chen, X., Yuan, L. L., Zhao, C., Birnbaum, S. G., Frick, A., Jung, W. E., ... Johnston, D. (2006). Deletion of Kv4.2 gene eliminates dendritic A-type K+ current and enhances induction of long-term potentiation in hippocampal CA1 pyramidal neurons. Journal of Neuroscience, 26, 12143-12151.

Cooper, L. N., \& Bear, M. F. (2012). The BCM theory of synapse modification at 30: Interaction of theory with experiment. Nature Reviews Neuroscience, 13, 798-810.

Cudmore, R. H., Fronzaroli-Molinieres, L., Giraud, P., \& Debanne, D. (2010). Spike-time precision and network synchrony are controlled by the homeostatic regulation of the D-type potassium current. Journal of Neuroscience, 30, 12885-12895.

Cudmore, R. H., \& Turrigiano, G. G. (2004). Long-term potentiation of intrinsic excitability in LV visual cortical neurons. Journal of Neurophysiology, 92, 341-348.

Daoudal, G., Hanada, Y., \& Debanne, D. (2002). Bidirectional plasticity of excitatory postsynaptic potential (EPSP)-spike coupling in CA1 hippocampal pyramidal neurons. Proceedings of the National Academy of Sciences of the United States of America, 99, 14512-14517.

Dasgupta, D., \& Sikdar, S. K. (2015). Calcium permeable AMPA receptor-dependent long lasting plasticity of intrinsic excitability in fast spiking interneurons of the dentate gyrus decreases inhibition in the granule cell layer. Hippocampus, 25, 269-285.

Dasgupta, D., \& Sikdar, S. K. (2019). Heterogeneous network dynamics in an excitatoryinhibitory network model by distinct intrinsic mechanisms in the fast spiking interneurons. Brain Research, 1714, 27-44.

Debanne, D., Gahwiler, B. H., \& Thompson, S. M. (1994). Asynchronous pre- and postsynaptic activity induces associative long-term depression in area CA1 of the rat hippocampus in vitro. Proceedings of the National Academy of Sciences of the United States of America, 91, 1148-1152.

Debanne, D., Gahwiler, B. H., \& Thompson, S. M. (1996). Cooperative interactions in the induction of long-term potentiation and depression of synaptic excitation between hippocampal CA3-CA1 cell pairs in vitro. Proceedings of the National Academy of Sciences of the United States of America, 93, 11225-11230.

Debanne, D., Gahwiler, B. H., \& Thompson, S. M. (1998). Long-term synaptic plasticity between pairs of individual CA3 pyramidal cells in rat hippocampal slice cultures. Journal of Physiology, 507(Pt 1), 237-247.

Debanne, D., Inglebert, Y., \& Russier, M. (2019). Plasticity of intrinsic neuronal excitability. Current Opinion in Neurobiology, 54, 73-82.

Dehorter, N., Ciceri, G., Bartolini, G., Lim, L., del Pino, I., \& Marin, O. (2015). Tuning of fast-spiking interneuron properties by an activity-dependent transcriptional switch. Science, 349, 1216-1220.

Desai, N. S., Casimiro, T. M., Gruber, S. M., \& Vanderklish, P. W. (2006). Early postnatal plasticity in neocortex of Fmr1 knockout mice. Journal of Neurophysiology, 96, 1734-1745.

Desai, N. S., Rutherford, L. C., \& Turrigiano, G. G. (1999b). Plasticity in the intrinsic excitability of cortical pyramidal neurons. Nature Neuroscience, 2, 515-520.

Desai, N. S., Rutherford, L. C., \& Turrigiano, G. G. (1999a). BDNF regulates the intrinsic excitability of cortical neurons. Learning \& Memory, 6, 284-291.

Disterhoft, J. F., Coulter, D. A., \& Alkon, D. L. (1986). Conditioning-specific membrane changes of rabbit hippocampal neurons measured in vitro. Proceedings of the National Academy of Sciences of the United States of America, 83, 2733-2737.

Dudek, S. M., \& Bear, M. F. (1992). Homosynaptic long-term depression in area CA1 of hippocampus and effects of N-methyl-D-aspartate receptor blockade. Proceedings of the National Academy of Sciences of the United States of America, 89, 4363-4367.

Evans, M. D., Sammons, R. P., Lebron, S., Dumitrescu, A. S., Watkins, T. B., Uebele, V. N., ... Grubb, M. S. (2013). Calcineurin signaling mediates activity-dependent relocation of the axon initial segment. Journal of Neuroscience, 33, 6950-6963.

Faber, E. S., Delaney, A. J., \& Sah, P. (2005). SK channels regulate excitatory synaptic transmission and plasticity in the lateral amygdala. Nature Neuroscience, 8, 635-641.

Fan, Y., Fricker, D., Brager, D. H., Chen, X., Lu, H. C., Chitwood, R. A., \& Johnston, D. (2005). Activity-dependent decrease of excitability in rat hippocampal neurons through increases in I(h). Nature Neuroscience, 8, 1542-1551.

Feldman, D. E. (2000). Timing-based LTP and LTD at vertical inputs to layer II/III pyramidal cells in rat barrel cortex. Neuron, 27, 45-56.

Feldman, D. E. (2012). The spike-timing dependence of plasticity. Neuron, 75, 556-571.

Frick, A., Magee, J., \& Johnston, D. (2004). LTP is accompanied by an enhanced local excitability of pyramidal neuron dendrites. Nature Neuroscience, 7, 126-135.

Gainey, M. A., Aman, J. W., \& Feldman, D. E. (2018). Rapid disinhibition by adjustment of PV intrinsic excitability during whisker map plasticity in mouse S1. Journal of Neuroscience, 38, 4749-4761.

Gasselin, C., Inglebert, Y., Ankri, N., \& Debanne, D. (2017). Plasticity of intrinsic excitability during LTD is mediated by bidirectional changes in h-channel activity. Scientific Reports, 7, 14418.

Gasselin, C., Inglebert, Y., \& Debanne, D. (2015). Homeostatic regulation of h-conductance controls intrinsic excitability and stabilizes the threshold for synaptic modification in CA1 neurons. Journal of Physiology, 593, 4855-4869.

Gastrein, P., Campanac, E., Gasselin, C., Cudmore, R. H., Bialowas, A., Carlier, E., .. Debanne, D. (2011). The role of hyperpolarization-activated cationic current in spiketime precision and intrinsic resonance in cortical neurons in vitro. Journal of Physiology, 589, 3753-3773.

Gustafsson, B., Wigstrom, H., Abraham, W. C., \& Huang, Y. Y. (1987). Long-term potentiation in the hippocampus using depolarizing current pulses as the conditioning stimulus to single volley synaptic potentials. Journal of Neuroscience, 7, 774-780.

Herring, B. E., \& Nicoll, R. A. (2016). Long-term potentiation: from CaMKII to AMPA receptor trafficking. Annual Review of Physiology, 78, 351-365.

Hoffman, D. A., \& Johnston, D. (1998). Downregulation of transient $\mathrm{K}+$ channels in dendrites of hippocampal CA1 pyramidal neurons by activation of PKA and PKC. Journal of Neuroscience, 18, 3521-3528.

Hoffman, D. A., Magee, J. C., Colbert, C. M., \& Johnston, D. (1997). K+ channel regulation of signal propagation in dendrites of hippocampal pyramidal neurons. Nature,
387, 869-875.

Huang, Y. Y., Kandel, E. R., Varshavsky, L., Brandon, E. P., Qi, M., Idzerda, R. L., .. Bourtchouladze, R. (1995). A genetic test of the effects of mutations in PKA on mossy fiber LTP and its relation to spatial and contextual learning. Cell, 83, 1211-1222.

Hull, C. A., Chu, Y., Thanawala, M., \& Regehr, W. G. (2013). Hyperpolarization induces a long-term increase in the spontaneous firing rate of cerebellar Golgi cells. Journal of Neuroscience, 33, 5895-5902.

Hyun, J. H., Eom, K., Lee, K. H., Bae, J. Y., Bae, Y. C., Kim, M. H., ... Lee, S. H. (2015). Kv1.2 mediates heterosynaptic modulation of direct cortical synaptic inputs in CA3 pyramidal cells. Journal of Physiology, 593, 3617-3643.

Jones, S. L., To, M. S., \& Stuart, G. J. (2017). Dendritic small conductance calcium-activated potassium channels activated by action potentials suppress EPSPs and gate spike-timing dependent synaptic plasticity. Elife, 6 .

Karmarkar, U. R., \& Buonomano, D. V. (2006). Different forms of homeostatic plasticity are engaged with distinct temporal profiles. European Journal of Neuroscience, 23, 1575-1584.

Kelso, S. R., Ganong, A. H., \& Brown, T. H. (1986). Hebbian synapses in hippocampus. Proceedings of the National Academy of Sciences of the United States of America, 83, 5326-5330.

Kim, J., Jung, S. C., Clemens, A. M., Petralia, R. S., \& Hoffman, D. A. (2007). Regulation of dendritic excitability by activity-dependent trafficking of the A-type $\mathrm{K}+$ channel subunit Kv4.2 in hippocampal neurons. Neuron, 54, 933-947.

Kim, H. H., Park, J. M., Lee, S. H., \& Ho, W. K. (2019). Association of mGluR-dependent LTD of excitatory synapses with endocannabinoid-dependent LTD of inhibitory synapses leads to EPSP to spike potentiation in CA1 pyramidal neurons. Journal of Neuroscience, 39, 224-237.

Kirchheim, F., Tinnes, S., Haas, C. A., Stegen, M., \& Wolfart, J. (2013). Regulation of action potential delays via voltage-gated potassium Kv1.1 channels in dentate granule cells during hippocampal epilepsy. Frontiers in Cellular Neuroscience, 7, 248.

Kirkwood, A., Dudek, S. M., Gold, J. T., Aizenman, C. D., \& Bear, M. F. (1993). Common forms of synaptic plasticity in the hippocampus and neocortex in vitro. Science, 260, 1518-1521.

Kuba, H., Yamada, R., Ishiguro, G., \& Adachi, R. (2015). Redistribution of Kv1 and Kv7 enhances neuronal excitability during structural axon initial segment plasticity. Nature Communications, 6, 8815.

Lamsa, K. P., Heeroma, J. H., Somogyi, P., Rusakov, D. A., \& Kullmann, D. M. (2007). Anti-Hebbian long-term potentiation in the hippocampal feedback inhibitory circuit. Science, 315, 1262-1266.

Larkum, M. E., Zhu, J. J., \& Sakmann, B. (2001). Dendritic mechanisms underlying the coupling of the dendritic with the axonal action potential initiation zone of adult rat layer 5 pyramidal neurons. Journal of Physiology, 533, 447-466.

Li, K. X., Lu, Y. M., Xu, Z. H., Zhang, J., Zhu, J. M., Zhang, J. M., ... Li, X. M. (2011). Neuregulin 1 regulates excitability of fast-spiking neurons through Kv1.1 and acts in epilepsy. Nature Neuroscience, 15, 267-273.

Lisman, J. (1989). A mechanism for the Hebb and the anti-Hebb processes underlying learning and memory. Proceedings of the National Academy of Sciences of the United States of America, 86, 9574-9578.

Lisman, J., Yasuda, R., \& Raghavachari, S. (2012). Mechanisms of CaMKII action in longterm potentiation. Nature Reviews Neuroscience, 13, 169-182.

Lu, Y. M., Mansuy, I. M., Kandel, E. R., \& Roder, J. (2000). Calcineurin-mediated LTD of GABAergic inhibition underlies the increased excitability of CA1 neurons associated with LTP. Neuron, 26, 197-205.

Maffei, A., \& Turrigiano, G. G. (2008). Multiple modes of network homeostasis in visual cortical layer 2/3. Journal of Neuroscience, 28, 4377-4384.

Magee, J. C. (1999). Dendritic lh normalizes temporal summation in hippocampal CA1 neurons. Nature Neuroscience, 2, 508-514.

Magee, J. C. (2000). Dendritic integration of excitatory synaptic input. Nature Reviews Neuroscience, 1, 181-190.

Mahon, S., \& Charpier, S. (2012). Bidirectional plasticity of intrinsic excitability controls sensory inputs efficiency in layer 5 barrel cortex neurons in vivo. Journal of Neuroscience, 32, 11377-11389.

Malenka, R. C. (1991). Postsynaptic factors control the duration of synaptic enhancement in area CA1 of the hippocampus. Neuron, 6, 53-60.

Markram, H., Lubke, J., Frotscher, M., \& Sakmann, B. (1997). Regulation of synaptic efficacy by coincidence of postsynaptic APs and EPSPs. Science, 275, 213-215.

Mellor, J., Nicoll, R. A., \& Schmitz, D. (2002). Mediation of hippocampal mossy fiber long-term potentiation by presynaptic Ih channels. Science, 295, 143-147.

Milshtein-Parush, H., Frere, S., Regev, L., Lahav, C., Benbenishty, A., Ben-Eliyahu, S., ... Slutsky, I. (2017). Sensory deprivation triggers synaptic and intrinsic plasticity in the hippocampus. Cerebral Cortex, 27, 3457-3470.

Mishra, P., \& Narayanan, R. (2015). High-conductance states and A-type K+ channels are potential regulators of the conductance-current balance triggered by HCN channels. Journal of Neurophysiology, 113, 23-43.

Misonou, H., Mohapatra, D. P., Park, E. W., Leung, V., Zhen, D., Misonou, K., ... Trimmer, J. S. (2004). Regulation of ion channel localization and phosphorylation by neuronal activity. Nature Neuroscience, 7, 711-718.

Mulkey, R. M., \& Malenka, R. C. (1992). Mechanisms underlying induction of homosynaptic long-term depression in area CA1 of the hippocampus. Neuron, 9, 967-975.

Nelson, A. B., Faulstich, M., Moghadam, S., Onori, K., Meredith, A., \& du Lac, S. (2017). BK channels are required for multisensory plasticity in the oculomotor system. Neuron, 93, 211-220.

Nelson, A. B., Krispel, C. M., Sekirnjak, C., \& du Lac, S. (2003). Long-lasting increases in intrinsic excitability triggered by inhibition. Neuron, 40, 609-620.

Ngo-Anh, T. J., Bloodgood, B. L., Lin, M., Sabatini, B. L., Maylie, J., \& Adelman, J. P. (2005). SK channels and NMDA receptors form a $\mathrm{Ca}^{2+}$-mediated feedback loop in dendritic spines. Nature Neuroscience, 8, 642-649. 
Oh, M. M., Kuo, A. G., Wu, W. W., Sametsky, E. A., \& Disterhoft, J. F. (2003). Watermaze learning enhances excitability of CA1 pyramidal neurons. Journal of Neurophysiology, 90, 2171-2179.

Ohtsuki, G., \& Hansel, C. (2018). Synaptic potential and plasticity of an SK2 channel gate regulate spike burst activity in cerebellar purkinje cells. iScience, 1, 49-54.

Ohtsuki, G., Piochon, C., Adelman, J. P., \& Hansel, C. (2012). SK2 channel modulation contributes to compartment-specific dendritic plasticity in cerebellar Purkinje cells. Neuron, 75, 108-120.

Orr, A. L., Hanson, J. E., Li, D., Klotz, A., Wright, S., Schenk, D., ... Madison, D. V. (2014). Beta-Amyloid inhibits E-S potentiation through suppression of cannabinoid receptor 1-dependent synaptic disinhibition. Neuron, 82, 1334-1345.

Paz, J. T., Mahon, S., Tiret, P., Genet, S., Delord, B., \& Charpier, S. (2009). Multiple forms of activity-dependent intrinsic plasticity in layer $\mathrm{V}$ cortical neurones in vivo. Journal of Physiology, 587, 3189-3205.

Penn, A. C., Zhang, C. L., Georges, F., Royer, L., Breillat, C., Hosy, E., ... Choquet, D. (2017). Hippocampal LTP and contextual learning require surface diffusion of AMPA receptors. Nature, 549, 384-388.

Raab-Graham, K. F., Haddick, P. C., Jan, Y. N., \& Jan, L. Y. (2006). Activity- and mTORdependent suppression of Kv1.1 channel mRNA translation in dendrites. Science, 314, 144-148.

Rama, S., Zbili, M., Fekete, A., Tapia, M., Benitez, M. J., Boumedine, N., ... Debanne, D. (2017). The role of axonal Kv1 channels in CA3 pyramidal cell excitability. Scientific Reports, 7, 315 .

Ross, S. T., \& Soltesz, I. (2001). Long-term plasticity in interneurons of the dentate gyrus. Proceedings of the National Academy of Sciences of the United States of America, 98 8874-8879.

Sacktor, T. C., \& Hell, J. W. (2017). The genetics of PKMzeta and memory maintenance. Science Signaling, 10

Santini, E., Quirk, G. J., \& Porter, J. T. (2008). Fear conditioning and extinction differentially modify the intrinsic excitability of infralimbic neurons. Journal of Neuroscience, 28, 4028-4036.

Santoro, B., Piskorowski, R. A., Pian, P., Hu, L., Liu, H., \& Siegelbaum, S. A. (2009). TRIP8b splice variants form a family of auxiliary subunits that regulate gating and trafficking of HCN channels in the brain. Neuron, 62, 802-813.

Sastry, B. R., Goh, J. W., \& Auyeung, A. (1986). Associative induction of posttetanic and long-term potentiation in CA1 neurons of rat hippocampus. Science, 232, 988-990.

Schreurs, B. G., Tomsic, D., Gusev, P. A., \& Alkon, D. L. (1997). Dendritic excitability microzones and occluded long-term depression after classical conditioning of the rabbit's nictitating membrane response. Journal of Neurophysiology, 77, 86-92.

Shah, M. M. (2014). Cortical HCN channels: Function, trafficking and plasticity. Journal of Physiology, 592, 2711-2719.

Shah, M. M., Anderson, A. E., Leung, V., Lin, X., \& Johnston, D. (2004). Seizure-induced plasticity of h channels in entorhinal cortical layer III pyramidal neurons. Neuron, 44, 495-508.

Shim, H. G., Jang, S. S., Jang, D. C., Jin, Y., Chang, W., Park, J. M., \& Kim, S. J. (2016). mGlu1 receptor mediates homeostatic control of intrinsic excitability through Ih in cerebellar Purkinje cells. Journal of Neurophysiology, 115, 2446-2455.

Shim, H. G., Jang, D. C., Lee, J., Chung, G., Lee, S., Kim, Y. G., ... Kim, S. J. (2017). Long term depression of intrinsic excitability accompanied by synaptic depression in cerebellar Purkinje cells. Journal of Neuroscience, 37, 5659-5669.

Sourdet, V., Russier, M., Daoudal, G., Ankri, N., \& Debanne, D. (2003). Long-term enhancement of neuronal excitability and temporal fidelity mediated by metabotropic glutamate receptor subtype 5. Journal of Neuroscience, 23, 10238-10248.

Spruston, N. (2008). Pyramidal neurons: Dendritic structure and synaptic integration. Nature Reviews Neuroscience, 9, 206-221.

Sun, Q. Q. (2009). Experience-dependent intrinsic plasticity in interneurons of barrel cortex layer IV. Journal of Neurophysiology, 102, 2955-2973.

Tang, S. J., Reis, G., Kang, H., Gingras, A. C., Sonenberg, N., \& Schuman, E. M. (2002). A rapamycin-sensitive signaling pathway contributes to long-term synaptic plasticity in the hippocampus. Proceedings of the National Academy of Sciences of the United States of America, 99, 467-472.

Tigaret, C. M., Chamberlain, S. E. L., Sadowski, J., Hall, J., Ashby, M. C., \& Mellor, J. R. (2018). Convergent metabotropic signaling pathways inhibit SK channels to promote synaptic plasticity in the hippocampus. Journal of Neuroscience, 38, 9252-9262.

Titley, H. K., Brunel, N., \& Hansel, C. (2017). Toward a neurocentric view of learning. Neuron, 95, 19-32.

Turrigiano, G. G., \& Nelson, S. B. (2004). Homeostatic plasticity in the developing nervous system. Nature Reviews Neuroscience, 5, 97-107.

Wang, Z., Xu, N. L., Wu, C. P., Duan, S., \& Poo, M. M. (2003). Bidirectional changes in spatial dendritic integration accompanying long-term synaptic modifications. Neuron, 37, 463-472.

Winder, D. G., \& Sweatt, J. D. (2001). Roles of serine/threonine phosphatases in hippocampal synaptic plasticity. Nature Reviews Neuroscience, 2, 461-474.

Xu, J., Kang, N., Jiang, L., Nedergaard, M., \& Kang, J. (2005). Activity-dependent longterm potentiation of intrinsic excitability in hippocampal CA1 pyramidal neurons. Journal of Neuroscience, 25, 1750-1760.

Zalutsky, R. A., \& Nicoll, R. A. (1990). Comparison of two forms of long-term potentiation in single hippocampal neurons. Science, 248, 1619-1624.

Zenke, F., \& Gerstner, W. (2017). Hebbian plasticity requires compensatory processes on multiple timescales. Philosophical Transactions of the Royal Society of London. Series B, Biological sciences, 372 .

Zhang, W., \& Linden, D. J. (2003). The other side of the engram: Experience-driven changes in neuronal intrinsic excitability. Nature Reviews Neuroscience, 4, 885-900. 